\title{
Plan de acciones inmediatas
}

Blanco Imeldo de Magaño

Viceministro de Comercio e Industria

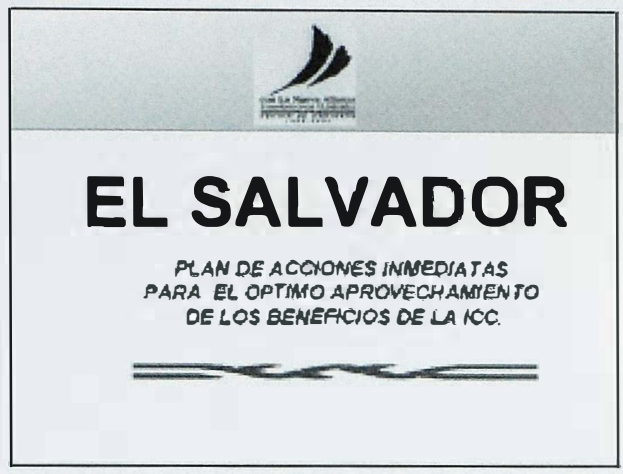

\begin{tabular}{|c|c|}
\hline \multicolumn{2}{|c|}{$\begin{array}{l}\text { OPORTUNIDADES: } \\
\text { IMPACTO ECONOMICO Y SOCIAL DE } \\
\text { BENEFICIOS DE LA ICC }\end{array}$} \\
\hline \multicolumn{2}{|c|}{$\checkmark$ CREACHON DE NUEVOS EMPLEOS } \\
\hline $\begin{array}{l}\text { Costura Directos } \\
\text { Castura Indineciós }\end{array}$ & $\begin{array}{l}60,000 \\
60,000\end{array}$ \\
\hline $\begin{array}{l}\text { Corte Dinectos } \\
\text { Teniche y Acabazato de Telas } \\
\text { Otros Indirectos }\end{array}$ & $\begin{array}{l}15,000 \\
15,000 \\
20,000\end{array}$ \\
\hline IOTAL & 150,000 \\
\hline
\end{tabular}

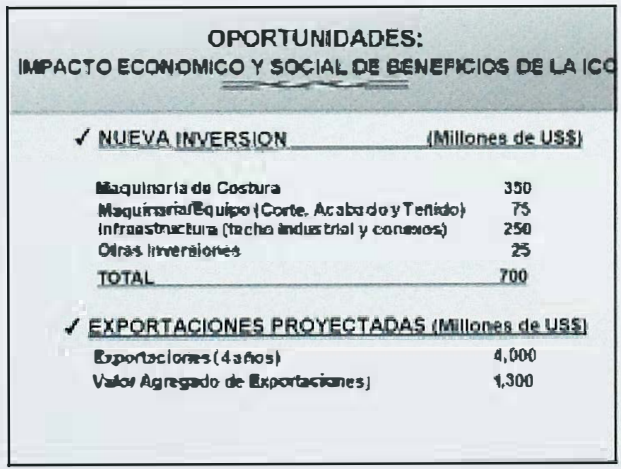



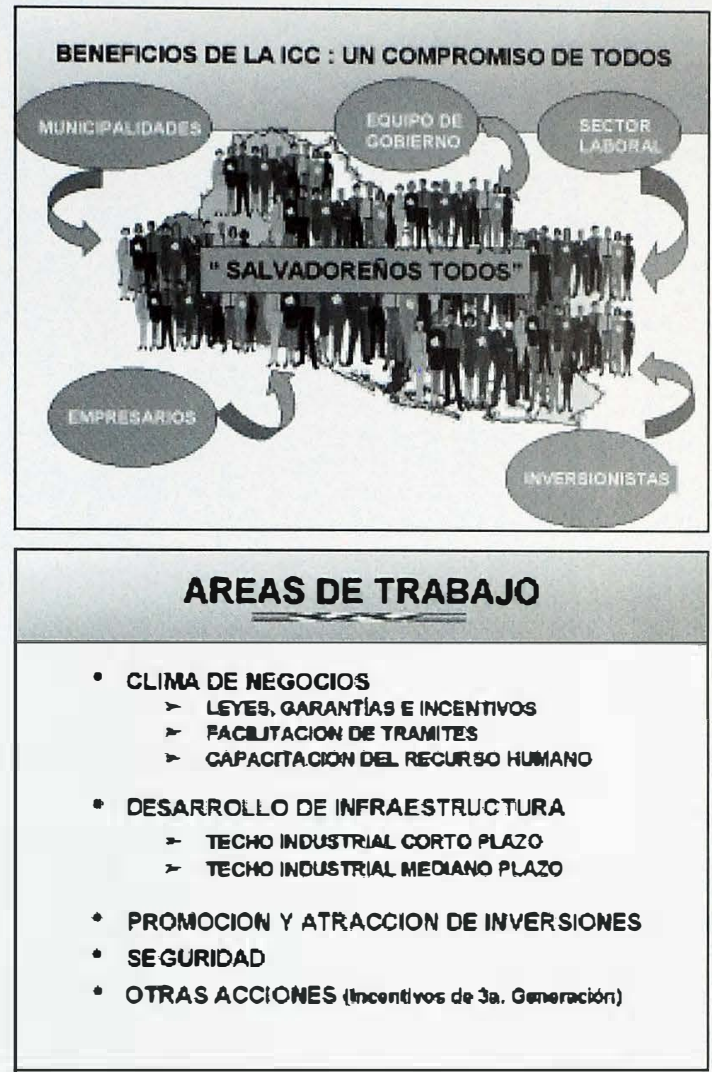

\section{CLIMA DE NEGOCIOS: LeYeS}

- Ley Especial de Reactivación del Empleo.

MIM.TFAB.

- Nueva Normativa de Aprendizaje.

- Mecanismos de Certificación de Condlciones Laborales.

MIN.TRAB.

MIN.TRAB.

- Mecaniamo privado de solución de controverslas laborales (previo a

MIN. TRAB. recurrir a instancias legales.

- Traducción Leyes de Inversión al Ingliss (Web siles) 


\section{CLIMA DE NEGOCIOS: LeYeS}

- Reformas a Ley de Zonas Francas:

MINEC.

- ncentivics eepectales plestableomiento

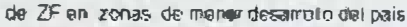
Secretaris

Requicitos bunteos de instalacion de induatrias Técnicaen serteres de teconoloplas de informagón Secretapla

- Tratamuenta expedno pera daneounés sin valer Legal comercial

- Adilear Feglamento de Ley zunas Francás

- Plan de Divulgación de Leyes

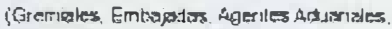
Despactos Legolea, AEArissar, etci!

\section{CLIMA DE NEGOCIOS: Facilitación de Trámites}

- Aglilzación Máxima de Trámites de Imversión (ONI).

- Nueva plataforma de Trámiles de Exportación a través de INTERNET.

- Agilización de Trámites Ambientales.

MINEC

- Aglizachón dé Trámiles Aduanales. (Retarzamento Teecegrazho!

- Consolidación de Aduanas Binacionales (EI Salvador-Ouatemala-Nic aragua)

\section{CENTREX/BCR}

MARN

MHDA'sector Empresarial.

MHDA'Seclor Empresarlai.

- Puesta en marcha de Ventanilla Unica de Trámiles de Construcción (mofi. vinnvarenca

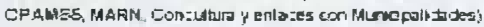

VM.Vivienda.

\section{CLIMA DE NEGOCIOS: Facilltaclón de Tramitas}

- Identificación y solución de limitantes en sector trans porte: Maritimo, Aér eo y Torrestie, 'Ante incremento valtumen de carazi)

VM Transporte CEPA Y Serctor Privado (Camara de Comerclo)

- Ordenamiento de servicios de taxis en Aeropuerto.

VMTransporte CEPA Privado (Cám. de Com)

- Ordenamiento de Transporte Público

VuTranspoite y Seclor Privado

- Corksolidar "Paso Fácil a Inver sionistas" en Aeropuerto. 


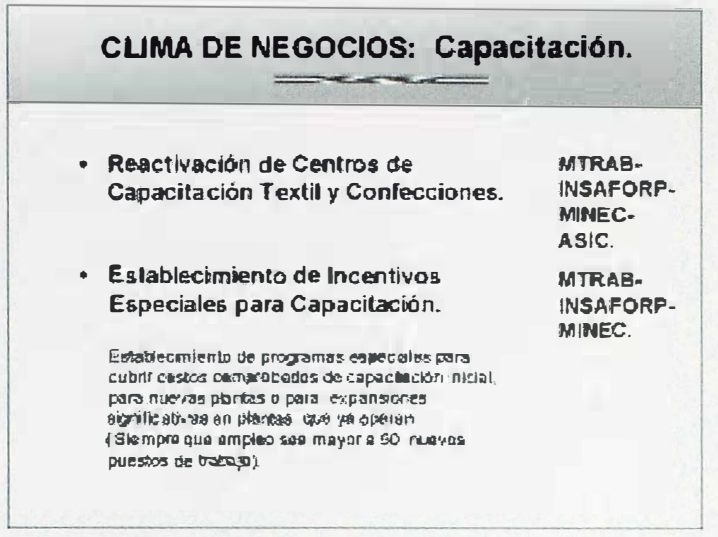

\begin{tabular}{|c|c|}
\hline \multicolumn{2}{|c|}{ DESARROLIO DE INFRAESTRUCTURA } \\
\hline $\begin{array}{l}\text { TECHO INDUSTRIAL-CORTO PLA } \\
\text { [Con serviclos publleos y t werzB loboral dispontble }\end{array}$ & ( a alrededores) \\
\hline $\begin{array}{l}\text { - Inventarlo de capacidad de expansión } \\
\text { en ZFs. y Parques Industriales operando. }\end{array}$ & MINEC \\
\hline $\begin{array}{l}\text { - Solución a obsiáculos de infraestructidra } \\
\text { de sen. pubblicos en ZF y Pls operando. }\end{array}$ & $\begin{array}{l}\text { MINEC-MOP. } \\
\text { ANOA-CORSAIM- } \\
\text { FISEL-VMNDU }\end{array}$ \\
\hline 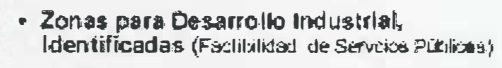 & $\begin{array}{l}\text { VANIVienda } \\
\text { MANEC }\end{array}$ \\
\hline 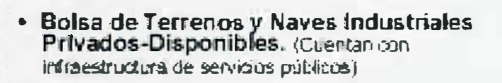 & MOP.VMViv. \\
\hline $\begin{array}{l}\text { - Fuentes de Financiamiento Competitivas } \\
\text { para desarrollo de techo industrial. }\end{array}$ & $\begin{array}{l}\text { BCRIBMIl } \\
\text { ABANSA } \\
\text { MINEC }\end{array}$ \\
\hline
\end{tabular}

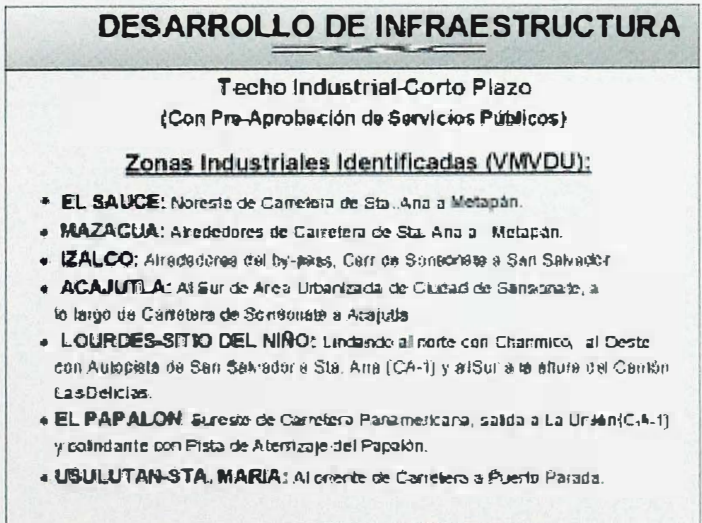




\begin{tabular}{|c|c|}
\hline \multicolumn{2}{|c|}{ DESARROLLO DE INFRAESTRUCTURA } \\
\hline \multicolumn{2}{|c|}{ Techo industrial - Mediano Plazo (Con Fuerca Laboral Disponible) } \\
\hline 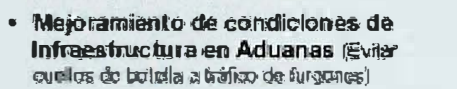 & MHOA I MOP \\
\hline $\begin{array}{l}\text { - Acelerar Consirucción de Puerto } \\
\text { Cutuca. }\end{array}$ & CEPA \\
\hline 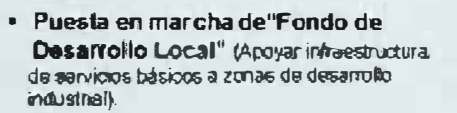 & $\begin{array}{l}\text { Fisousecrelaria } \\
\text { Teenita. }\end{array}$ \\
\hline $\begin{array}{l}\text { - Acelerar Inicio Operaciones de Ferry. } \\
\text { (La Unión) }\end{array}$ & CEPAMMRREE \\
\hline
\end{tabular}

mopisecrotaris Techica-Presid. Red Vial Competitiva.

MOPI Secsetarla Técnicafiesid. Caminos Rurales.

- Desarrallos habitaclonales. (Planificar en función de incremento de demends de convicins slrededor de arnolio industrial)
MOP / VMNOU 
Medidas Inmediatas

Ia ampliación de la iniciativa de la cuenca del caribe

\section{PROMOCION Y ATRACCION DE INVERSIONES}

- Reposileionamiento ontemacionsl de El Sevador (Post-Confilicto)

- Presentación EI Nuevo El Salvador" ente comunidad Infernaclonal de inversloneas.

- Uso de estrategias especiallzadas pl atroción de inversiones

- Participación en eventas promocionales espocializados (Estados Unidos y Ásia).

- Ejecución intenelva de Programas Promocionales de Inversion y Codinver andin.

- Miabones focalleadas a palises con potancial de inversion.

\section{SEGURIDAD}

- Refornas logales e Institucionales que garantucen la Segurldad Crudedana

- Ejecución Intenarva de Programas Especlales de Segurlded Cludadana.

\section{Nacional} de Promoción de Inversiones.

(APOYO MINEC Y MRREE।
- Nuevos mecanismos de Protección al Trensita do Miercancias ifveras

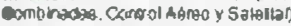

- Establecimiento de Córredores de Sequridad

Msogurdad Pübicarives MDefensa MHuclenda

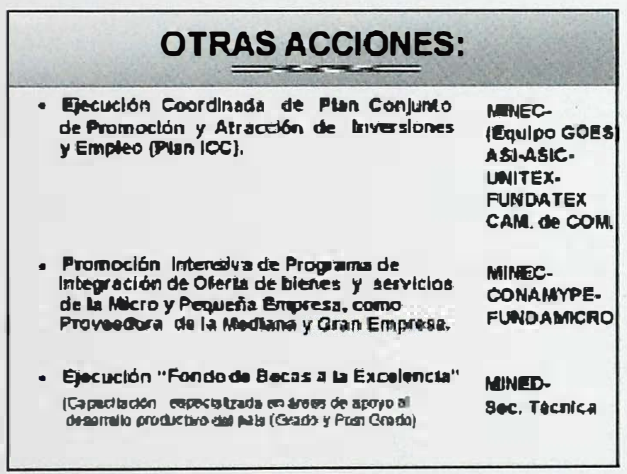

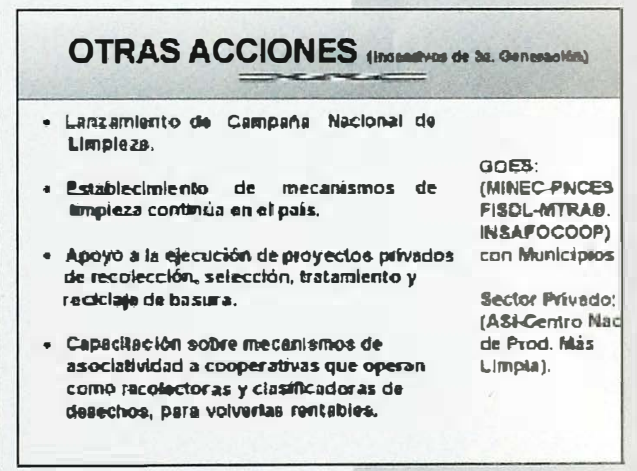

